\title{
Association between Homocysteine Levels and Psoriasis: A Meta-Analysis
}

\author{
Jung Eun Kim, Ho Jung Lee, Jong Suk Lee, Kyu Uang Whang ${ }^{1}$, Young Lip Park ${ }^{2}$, Sung Yul Lee, \\ Hyun Jung $\mathrm{Kim}^{3}$
}

Department of Dermatology, Soonchunhyang University Cheonan Hospital, Cheonan, ${ }^{1}$ Department of Dermatology, Soonchunhyang University Seoul Hospital, Seoul, ${ }^{2}$ Department of Dermatology, Soonchunhyang University Bucheon Hospital, Bucheon, ${ }^{3}$ Department of Preventive Medicine, Korea University College of Medicine, Seoul, Korea

Background: Psoriasis is a multifactorial disease associated with an increased risk for metabolic syndrome and cardiovascular diseases. Elevated levels of homocysteine (Hcy) are a marker of cardiovascular risk. Several studies have evaluated the associations between psoriasis and Hcy levels; however, the results remain inconclusive. Objective: We performed a systematic review of the literature and a meta-analysis to better understand the relationship between psoriasis and Hcy. Methods: Five scientific databases (MEDLINE, Embase, Cochrane Library, Scopus, and Web of Science) were searched to identify relevant studies. A review of 307 publications identified 16 studies that directly assessed plasma levels of Hcy in psoriasis patients. Results: A total of 16 studies including 2,091 subjects were included in the meta-analysis. Hcy levels were significantly higher in psoriasis patients relative to healthy controls (weighted mean difference [WMD], 3.30; 95\% confidence interval [Cl], 1.58 $5.02 ; I^{2}=82.1 \%$ ). Subgroup analyses revealed that patients

Received May 24, 2018, Revised December 10, 2018, Accepted for publication December 17, 2018

Corresponding author: Sung Yul Lee, Department of Dermatology, Soonchunhyang University Cheonan Hospital, 31 Suncheonhyang 6-gil, Dongnam-gu, Cheonan 31151, Korea. Tel: 82-41-570-2270, Fax: 82-41-570-2271, E-mail: dermsung@schmc.ac.kr

ORCID: https://orcid.org/0000-0002-6995-4561

Hyun Jung Kim, Department of Preventive Medicine, Korea University College of Medicine, 145 Anam-ro, Seongbuk-gu, Seoul 02841, Korea. Tel: 82-2-2286-1351, Fax: 82-2-927-7220, E-mail: moole02@naver.com ORCID: https://orcid.org/0000-0003-2018-2385

This is an Open Access article distributed under the terms of the Creative Commons Attribution Non-Commercial License (http://creativecommons. org/licenses/by-nc/4.0) which permits unrestricted non-commercial use, distribution, and reproduction in any medium, provided the original work is properly cited.

Copyright (c) The Korean Dermatological Association and The Korean Society for Investigative Dermatology with higher mean psoriasis area severity index (PASI) scores (PASI > 10) had significantly higher Hcy levels compared to healthy controls (WMD, 4.17; 95\% Cl, 1.18 7.16; $I^{2}=88.3 \%$ ), whereas patients with lower mean PASI scores $(\mathrm{PASI} \leq 10)$ had not $(\mathrm{WMD}, 0.76 ; 95 \% \mathrm{Cl},-1.84 \sim 3.35$; $\left.I^{2}=72.2 \%\right)$. Conclusion: This meta-analysis found that psoriasis patients, in particular those with PASI $>10$, had significantly higher Hcy levels compared to healthy controls. Further research is needed to determine the association between Hcy levels and psoriasis severity. (Ann Dermatol 31(4) $378 \sim 386,2019$ )

\section{-Keywords-}

Homocysteine, Meta-analysis, Psoriasis

\section{INTRODUCTION}

Psoriasis is a common chronic inflammatory disease of the skin affecting $2 \%$ to $3 \%$ of the general population, with varying prevalence among different ethnic groups ${ }^{1}$. The characteristic skin lesion is a sharply demarcated erythematous scaly plaque, with evidence of abnormal epidermal thickening and inflammatory cell infiltrates, predominated by $\mathrm{T}$ lymphocytes. Recent advances in immunology and genetics have significantly altered our understanding of the disease, with many now regarding psoriasis as a systemic inflammatory disease, as opposed to a localized skin condition. Although psoriasis primarily manifests at the skin, recent studies have identified several serious comorbidities associated with this disease, including atherosclerosis, chronic ischemic heart disease, diabetes mellitus, and metabolic syndrome $\mathrm{e}^{2,3}$. As understanding of the pathogenesis of psoriasis has evolved, dysregulation of the im- 
mune system in disease pathology has taken center stage, characterized by systemic inflammatory processes resulting from the release of inflammatory cytokines, including tumor necrosis factor- $\alpha$, interleukin (IL)-17, and IL-22 4 . As these inflammation processes also play an important role in the pathogenesis of other diseases including atherosclerosis, cardiovascular diseases (CVDs), and metabolic syndrome, it is not surprising that many of these conditions are more common in psoriatic patients than in the general population ${ }^{5}$. Moreover, cardiovascular risk factors including diabetes, hypertension, hyperlipidemia, and obesity have been revealed to be more strongly associated with severe psoriasis than with mild psoriasis ${ }^{1,6,7}$. Accordingly, recent studies have sought to assess systemic inflammation in psoriasis using inflammatory biomarkers to measure psoriasis disease severity and to explain its comorbidities $^{8,9}$.

Homocysteine (Hcy) is a sulfur-containing amino acid that is generated as a result of the metabolism of methionine. The metabolism of Hcy occurs through two biochemical pathways; remethylation to methionine or conversion to cysteine via coenzymes such as vitamin B6, vitamin B12 and folic acid ${ }^{10,11}$.

The plasma Hcy level can be influenced by genetic factors, nutrition, chronic diseases and drugs. It was reported that hyperhomocysteinemia, an elevated plasma total Hcy concentration (the normal range is 5 to $15 \mu \mathrm{mol} / \mathrm{L}$ ) is related to the occurrence and development of many diseases, for example, atherosclerotic CVDs, stroke, peripheral arterial occlusive disease, and venous thrombosis ${ }^{12,13}$, although the underlying mechanisms responsible for these associations are only partially understood. Among the prevailing theories, a leading hypothesis is that Hcy can increase oxidative stress, resulting in increased endothelial dysfunction $^{5,14}$.

Several studies reported that patients with psoriasis have elevated levels of Hcy in the plasma in combination with lower levels of folate ${ }^{15-18}$. However, the correlation between Hcy levels and the severity of psoriasis remains controversial, with some studies having demonstrated a direct proportional relationship between them. Additional correlations have also been seen between Hcy levels and psoriasis duration ${ }^{10,13,14}$. To address this apparent divergence among published studies, we performed a systematic review of the literature and a meta-analysis to assess plasma levels of Hcy in patients with psoriasis, and their relationship to disease severity. We compared Hcy levels between psoriasis patients and controls, and tried to evaluate the correlation between Hcy levels and severity of psoriasis.

\section{MATERIALS AND METHODS}

This study was performed according to the Preferred Reporting Items for Systematic Reviews and Meta-Analysis (PRISMA) guidelines (Supplementary Table 1) ${ }^{19}$.

\section{Search strategy}

A systematic search of the literature was performed using five major scientific databases (MEDLINE, Embase, Cochrane Library, Scopus, and Web of Science) up to June 2017 to assess the potential association between Hcy levels and psoriasis. The search was not restricted based on language or publication year. The following keywords and medical subject headings were used for the MEDLINE search: "psoriasis" and "homocysteine." Search strategies for the other databases were developed based on the MEDLINE strategy (Supplementary Table 2). In addition to the initial electronic search, a manual search for additional relevant publications was also performed.

\section{Study selection}

Two reviewers independently assessed the eligible of each study for this meta-analysis using the inclusion and exclusion criteria. The study inclusion criteria consisted of the following: 1) studies evaluating the association between plasma levels of Hcy and psoriasis; 2) Hcy levels of psoriasis were presented as means with either standard deviations or standard errors; and 3) availability of a full-text article. Study exclusion criteria consisted of the following: 1) reviews and case reports with fewer than 10 subjects; and 2) studies without control groups. Final 16 studies ${ }^{5,12 \text {, }}$ $13,15-17,20-29$ were selected for further analysis.

\section{Data extraction}

Two reviewers independently extracted data from the 16 studies using a predefined data extraction form. Disagreements over data extraction were resolved by consensus. The following variables were assessed for each study: authors, year of publication, demographic characteristics of the study population (country, age, sex, and number), inclusion criteria for psoriasis, the plasma levels of Hcy in both the case and control groups, and psoriasis area severity index (PASI) score.

\section{Statistical analyses}

Hcy levels were compared using weighted mean differences (WMDs) and 95\% confidence interval $(\mathrm{Cl})$ and are presented as $\mu \mathrm{mol} / \mathrm{L}$. Heterogeneity was assessed using the $I^{2}$ static, which indicates the proportion of variation across trials attributable to heterogeneity, rather than sampling error. $l^{2}$ values $>50 \%$ and $p$-values from the $\chi^{2}$ test 
$<0.10$ were considered indicative of significant heterogeneity among the included studies. If substantial statistical heterogeneity was noted $\left(I^{2}>50 \%\right)$, a more detailed assessment of individual study characteristics and subgroups was planned to better understand the main body of evidence. The results were presented in a forest plot. In addition, potential publication bias was assessed by funnel plot and Egger test. Moreover, we also performed "trim and fill" analysis to further assess the possible influence of unpublished studies on the results of our meta-analysis. All calculations were performed using Stata MP version 13.0 (STATA Corp., College Station, TX, USA) and a $p$-value less than 0.05 indicated a significant difference. This study is based on Cochrane Review Methods.

\section{Quality assessment and sensitivity analysis}

The quality assessment was performed independently by two reviewer, using the Quality In Prognosis Studies (QUIPS) tool to assess the methodological quality of included stud$\mathrm{ies}^{30}$. The following six domains were assessed and recorded in all included studies: study participation; study attrition; prognostic factor measurement; outcome measurement; study confounding; and statistical analysis and reporting. Risk of bias in each of these six domains was categorized as 'low', 'moderate', or 'high'. We considered "study confounding" as an important bias domain in this study and thus, sensitivity analyses was done by the "study confounding" bias domain status.

\section{RESULTS}

\section{Identification of studies}

The database search yielded 484 articles. After excluding duplicates and studies that are not eligible according to in- clusion and exclusion criteria, 23 articles remained. Of those, seven studies were excluded after detailed assessment of the full text because they did not provide sufficient data (Fig. 1). The last remaining 16 studies were included in the meta-analysis.

\section{Study characteristics and patients}

All sixteen studies finally included for the meta-analysis were cross-sectional observational studies. Sixteen studies were comprised of a total of 2,091 participants, which included 1,172 patients with psoriasis and 919 healthy or hospital-based patients with other mild skin diseases. The sample sizes ranged from 13 to 200 psoriasis patients. All studies assessed and defined psoriasis using specific tools or by measuring clinical findings. With the exception of fives studies ${ }^{13,15,17,21,29}$, all used the PASI scoring system to evaluate psoriasis severity. The range of the mean Hcy levels was 11 to $24.73 \mu \mathrm{mol} / \mathrm{L}$ in psoriasis patients and 9.99 to $17.3 \mu \mathrm{mol} / \mathrm{L}$ in controls. Of the 16 included studies, 8 were carried out in Turkey, 4 in Italy, 2 in Spain, 1 in Norway, and 1 in Malaysia. The main characteristics of the studies are shown in Table $1^{5,12,13,15-17,20-29}$.

\section{Plasma levels of homocysteine and psoriasis}

This meta-analysis demonstrated that Hcy levels were significantly higher in psoriasis patients relative to controls (WMD， 3.30; 95\% Cl, 1.58 5.02; $I^{2}=82.1 \%$; Fig. $2 \mathrm{~A})^{5,12,13,15-17,20-28}$. Subgroup analyses showed that patients with higher mean PASI scores (PASI > 10) had significantly higher Hcy levels compared to healthy controls (WMD, 4.17; 95\% Cl, 1.18 7.16; $I^{2}=88.3 \%$ ), whereas patients with lower mean PASI scores (PASI $\leq 10)$ had not $(\mathrm{WMD}$, $0.76 ; 95 \% \mathrm{Cl},-1.84 \sim 3.35 ; I^{2}=72.2 \%$; Fig. $\left.2 \mathrm{~B}\right)^{5,12,16,20,22-28}$. In addition, there were two studies ${ }^{12,29}$ which individually

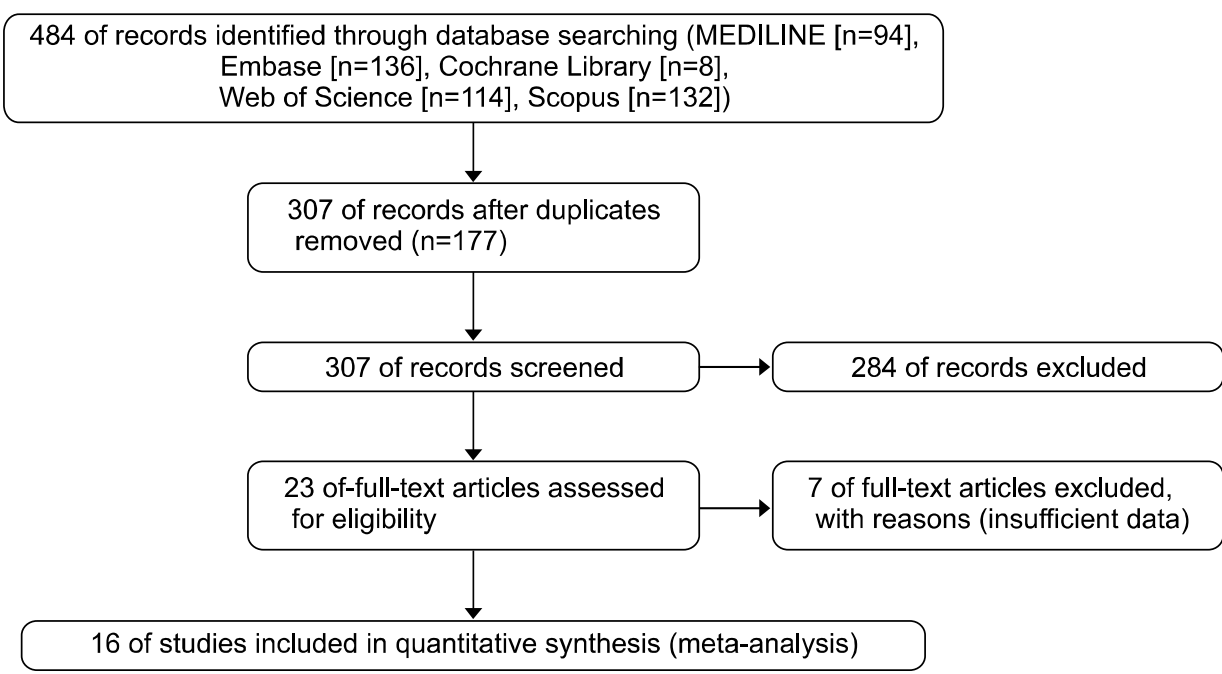

Fig. 1. Flow diagram of study identification, inclusion, and exclusion. 
Table 1. Characteristics of the 16 studies included in the final analysis

\begin{tabular}{|c|c|c|c|c|c|c|}
\hline \multirow[b]{2}{*}{ Study } & \multicolumn{2}{|c|}{ No. of patient } & \multirow[b]{2}{*}{ Age (yr) } & \multirow[b]{2}{*}{ PASI score } & \multicolumn{2}{|c|}{ Plasma homocysteine level } \\
\hline & $\begin{array}{l}\text { Psoriasis group } \\
\qquad(\mathrm{n}=1,172)\end{array}$ & $\begin{array}{l}\text { Control group } \\
\qquad(n=919)\end{array}$ & & & $\begin{array}{l}\text { Psoriasis group } \\
\qquad(n=1,172)\end{array}$ & $\begin{array}{l}\text { Control group } \\
\qquad(n=919)\end{array}$ \\
\hline Akcali et al., $2014^{26}$ & 50 & 40 & $\geq 18$ & $20.32 \pm 5.9$ & $13.64 \pm 7.97$ & $13.8 \pm 9.77$ \\
\hline Ataseven et al., $2014^{27}$ & 56 & 33 & $\geq 18$ & $8.3 \pm 6.5$ & $13.55 \pm 8.3$ & $16.04 \pm 9.3$ \\
\hline Bilgiç et al., $2015^{28}$ & 42 & 48 & $\geq 18$ & $10.64 \pm 6.77$ & $24.73 \pm 12.96$ & $17.3 \pm 7.65$ \\
\hline Brazzelli et al., $2010^{12}$ & 98 & 98 & - & $19.51 \pm 16.26$ & $19.6 \pm 15.1$ & $13.7 \pm 5.56$ \\
\hline Cakmak et al., $2009^{21}$ & 70 & 70 & $18 \sim 60$ & - & $16.76 \pm 16.62$ & $12.86 \pm 6.45$ \\
\hline Curcó et al., $2018^{29}$ & 178 & - & $\geq 20$ & - & $11 \pm 12$ & - \\
\hline Dogan and Atakan, $2010^{22}$ & 45 & 45 & - & $22.44 \pm 9.28$ & $12.64 \pm 5.54$ & $9.99 \pm 2.88$ \\
\hline Erturan et al., $2014^{5}$ & 56 & 53 & $\geq 18$ & $6.65 \pm 6.7$ & $14.33 \pm 7.61$ & $14.96 \pm 8.81$ \\
\hline Giannoni et al., $2015^{13}$ & 52 & 24 & - & - & $19.71 \pm 11.16$ & $13.9 \pm 11.2$ \\
\hline Gisondi et al., $2010^{23}$ & 172 & 198 & $\geq 18$ & $10.3 \pm 9.3$ & $16.3 \pm 12.7$ & $10.3 \pm 4.6$ \\
\hline Karabudak et al., $2008^{20}$ & 20 & 20 & - & $13 \pm 7$ & $21 \pm 8$ & $11 \pm 2$ \\
\hline Vanizor Kural et al., $2003^{16}$ & 30 & 30 & - & $5.52 \pm 3.63$ & $15.91 \pm 6.18$ & $11.36 \pm 4.46$ \\
\hline Liew et al., $2012^{24}$ & 200 & 167 & - & $8.92 \pm 7.64$ & $15.67 \pm 4.37$ & $14.89 \pm 3.69$ \\
\hline Malerba et al., $2006^{15}$ & 40 & 30 & $\geq 18$ & - & $16 \pm 5.6$ & $10.4 \pm 4.7$ \\
\hline Refsum et al., $1989^{17}$ & 13 & 13 & - & - & $14.4 \pm 4.8$ & $10.8 \pm 2.9$ \\
\hline Romaní et al., $2012^{25}$ & 50 & 50 & $\geq 18$ & $15.6 \pm 5.4$ & $12.1 \pm 5.1$ & $13.7 \pm 5.4$ \\
\hline
\end{tabular}

Values are presented as number only or mean \pm standard deviation. PASI: psoriasis area severity index, -: data not collected.

recorded Hcy levels in two groups of patients which was divided based on psoriasis severity; mild psoriasis and moderate-severe psoriasis. One study considered a moderate-severe psoriasis as a PASI score of 10 or more ${ }^{12}$. The other study defined it when PASI $>10$ and/or body surface area $>10$ or systemic treatment of psoriasis were performed $^{29}$. Meta-analysis of these two studies showed that patients with moderate-severe psoriasis had significantly higher Hcy levels compared to that of patients with mild disease (WMD, 4.50; 95\% Cl, 1.30 7.70; $I^{2}=0.0 \%$; Fig. $2 \mathrm{C})^{12,29}$. The characteristics of these two studies are shown in Table $2^{12,29}$.

\section{Quality assessment}

The six QUIPS domains with risk of bias for each of the 16 included studies are shown in Table 3,12,13,15-17,20-29. "Study confounding" was thought to be the most important bias domain in this study. As for this domain, six studies were considered at low risk of bias, three studies at moderate risk of bias, and seven studies at high risk of bias.

\section{Sensitivity analysis}

We conducted sensitivity analysis among studies showing low risk of bias at "study confounding" QUIPS domain. We individually analyzed studies according to the mean PASI score, thus we excluded one study ${ }^{15}$ not presenting mean PASI score. The sensitivity analysis outcomes are shown in Fig. $3^{5,20,24,27,28}$. Sensitivity analysis outcomes of two studies with higher mean PASI scores (PASI > 10) had significantly higher Hcy levels (WMD, 8.99; 95\% Cl, 6.17 $\sim 11.80 ; I^{2}=0 \%$; Fig. 3). However, three studies with lower PASI scores $(\mathrm{PASI}<10)$ havent' shown significantly higher Hcy levels.

\section{Publication bias}

Although visual inspection of the funnel plot suggested some asymmetry (Fig. 4), the result of Egger test did not reveal any significant publication bias $(p=0.37)$. Additional trim and fill analysis indicated that two more studies would be required to produce a symmetrical funnel plot. However, even after adjustment of effect size for potential publication bias, the corrected analysis continued to show a statistically higher serum Hcy levels in psoriasis patients relative to healthy controls (WMD, 2.56; 95\% Cl, 0.79 4.33). Thus, these results indicate there might be a publication bias, but it is unlikely that publication bias poses a significant threat to the current meta-analysis.

\section{DISCUSSION}

The results of our meta-analysis demonstrated that patients with psoriasis had higher plasma levels of Hcy than healthy controls, which was significant only in studies with high PASI scores. So far, several studies have examined Hcy levels in psoriasis patients, with most observing hyperhomocysteinemia in these patients. However, the role of Hcy in the pathophysiology of psoriasis and the exact mechanism causing hyperhomocysteinemia in psoriasis 
A Study ID

Akcali et al., $2014^{26}$

Ataseven et al., $2014^{27}$

Bilgiç et al., $2015^{28}$

Brazzelli et al., $2010^{12}$

Cakmak et al., $2009^{21}$

Dogan and Atakan, $2010^{22}$

Erturan et al., $2014^{5}$

Giannoni et al., $2011^{13}$

Gisondi et al., $2010^{23}$

Karabudak et al., $2008^{20}$

Vanzinor Kural et al., $2003^{16}$

Liew et al., $2012^{24}$

Malerba et al., $2006^{15}$

Refsum et al., $1989^{17}$

Romaní et al., $2012^{25}$

Overall (I-squared $=82.1 \%, p=0.000$ )

Note: Weights are from random effects analysis.

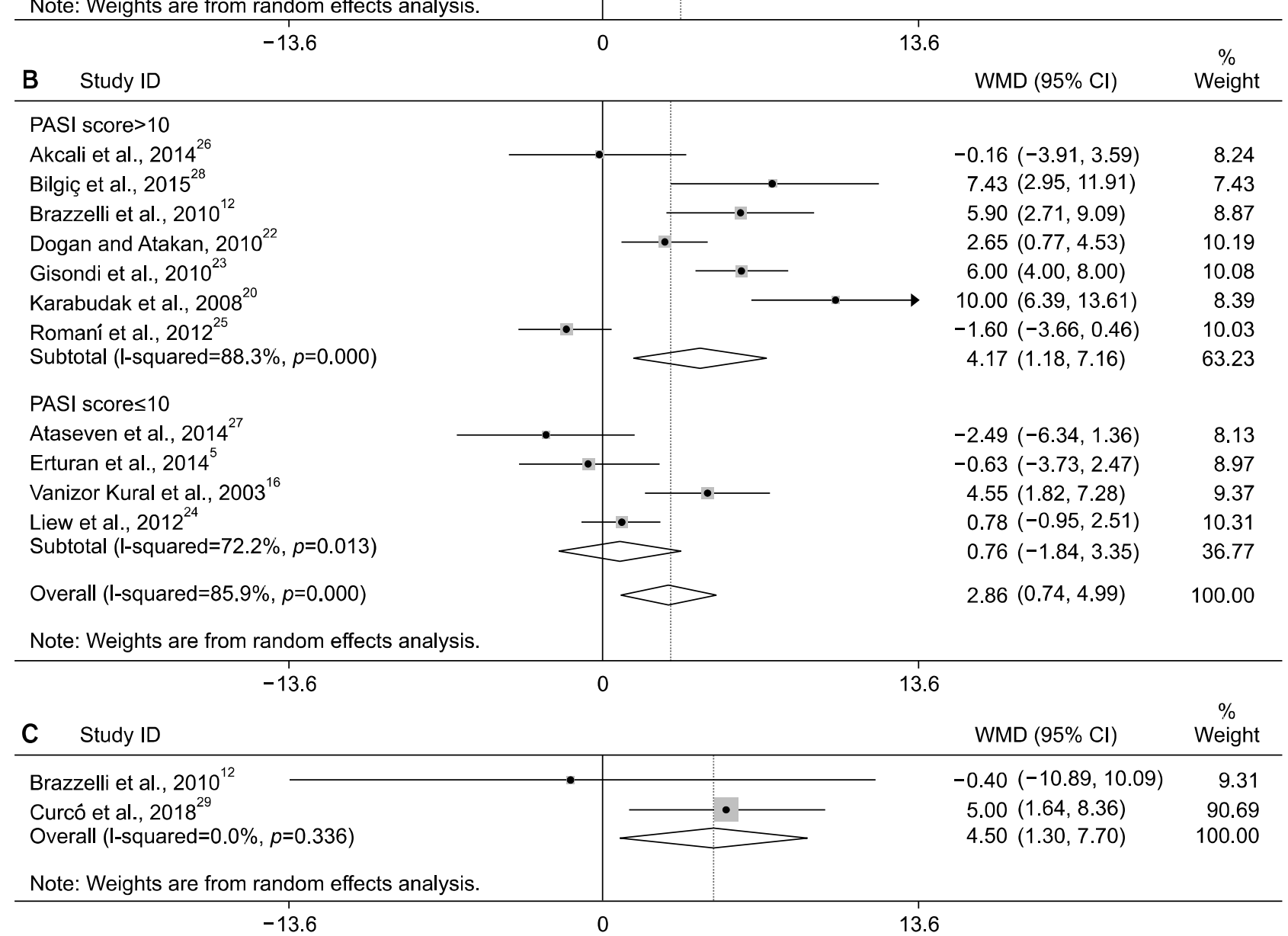

Fig. 2. Forest plot of the meta-analysis for homocysteine levels. (A) Homocysteine levels in psoriasis patients and controls. (B) Subgroup analysis according to mean psoriasis area severity index (PASI) scores. (C) Meta-analysis of two studies which directly compared homocysteine levels according to psoriasis severity within studies. WMD: weighted mean difference, Cl: confidence interval.

has not been fully established.

Psoriasis is a chronic inflammatory skin disease with complex pathophysiology. The inflammatory reactions in psor- iasis may be associated with increased reactive oxygen species. Oxidative stress, an imbalance between oxidants and antioxidants, can disrupt redox signaling and lead mo- 
Table 2. Characteristics of the 2 studies separately evaluated homocysteine (Hcy) levels based on psoriasis severity

\begin{tabular}{lcccccc}
\hline \multirow{2}{*}{ Study } & \multicolumn{2}{c}{ No. of patient } & & \multicolumn{2}{c}{ Plasma Hcy level } \\
\cline { 2 - 3 } \cline { 6 - 6 } & $\begin{array}{c}\text { Mild psoriasis } \\
(\mathrm{n}=115)\end{array}$ & $\begin{array}{c}\text { Moderate-severe psoriasis } \\
(\mathrm{n}=161)\end{array}$ & $\begin{array}{c}\text { Mild psoriasis } \\
(\mathrm{n}=115)\end{array}$ & $\begin{array}{c}\text { Moderate-severe psoriasis } \\
(\mathrm{n}=161)\end{array}$ \\
\hline Brazzelli et al., $2010^{12}$ & 16 & 82 & & $19.9 \pm 20.5$ & $19.5 \pm 13.9$ \\
Curcó et al., $2018^{29}$ & 99 & 79 & $9 \pm 3$ & $14 \pm 18$ \\
\hline
\end{tabular}

Values are presented as number only or mean \pm standard deviation.

Table 3. Quality assessment of included studies (risk of bias in six different domains according to the Quality In Prognosis Studies [QUIPS] tool [Hayden et al, $2013^{30}$ ])

\begin{tabular}{|c|c|c|c|c|c|c|}
\hline Study & $\begin{array}{c}\text { Study } \\
\text { participation }\end{array}$ & Study attrition & $\begin{array}{c}\text { Prognostic factor } \\
\text { measurement }\end{array}$ & $\begin{array}{c}\text { Outcome } \\
\text { measurement }\end{array}$ & $\begin{array}{c}\text { Study } \\
\text { confounding }\end{array}$ & $\begin{array}{c}\text { Statistical } \\
\text { analysis }\end{array}$ \\
\hline Akcali et al., $2014^{26}$ & Low & Low & Low & Low & High & Low \\
\hline Ataseven et al., $2014^{27}$ & Low & Low & Low & Low & Low & Low \\
\hline Bilgiç et al., $2015^{28}$ & Low & Low & Low & Low & Low & Low \\
\hline Brazzelli et al., $2010^{12}$ & Low & Low & Low & Low & Moderate & Low \\
\hline Cakmak et al., $2009^{21}$ & Low & Low & Low & Low & Moderate & Low \\
\hline Curcó et al., $2018^{29}$ & Low & Low & Low & Low & High & Low \\
\hline Dogan and Atakan, $2010^{22}$ & Low & Low & Low & Low & High & Low \\
\hline Erturan et al., $2014^{5}$ & Low & Low & Low & Low & Low & Low \\
\hline Giannoni et al., $2015^{13}$ & Low & Low & Low & Low & High & Low \\
\hline Gisondi et al., $2010^{23}$ & Low & Low & Low & Low & Moderate & Low \\
\hline Karabudak et al., $2008^{20}$ & Low & Low & Low & Low & Low & Low \\
\hline Vanizor Kural et al., $2003^{16}$ & Low & Low & Low & Low & High & Low \\
\hline Liew et al., $2012^{24}$ & Low & Low & Low & Low & Low & Low \\
\hline Malerba et al., $2006^{15}$ & Low & Low & Low & Low & Low & Low \\
\hline Refsum et al., $1989^{17}$ & Low & Low & Low & Low & High & Low \\
\hline Romaní et al., $2012^{25}$ & Low & Low & Low & Low & High & Low \\
\hline
\end{tabular}

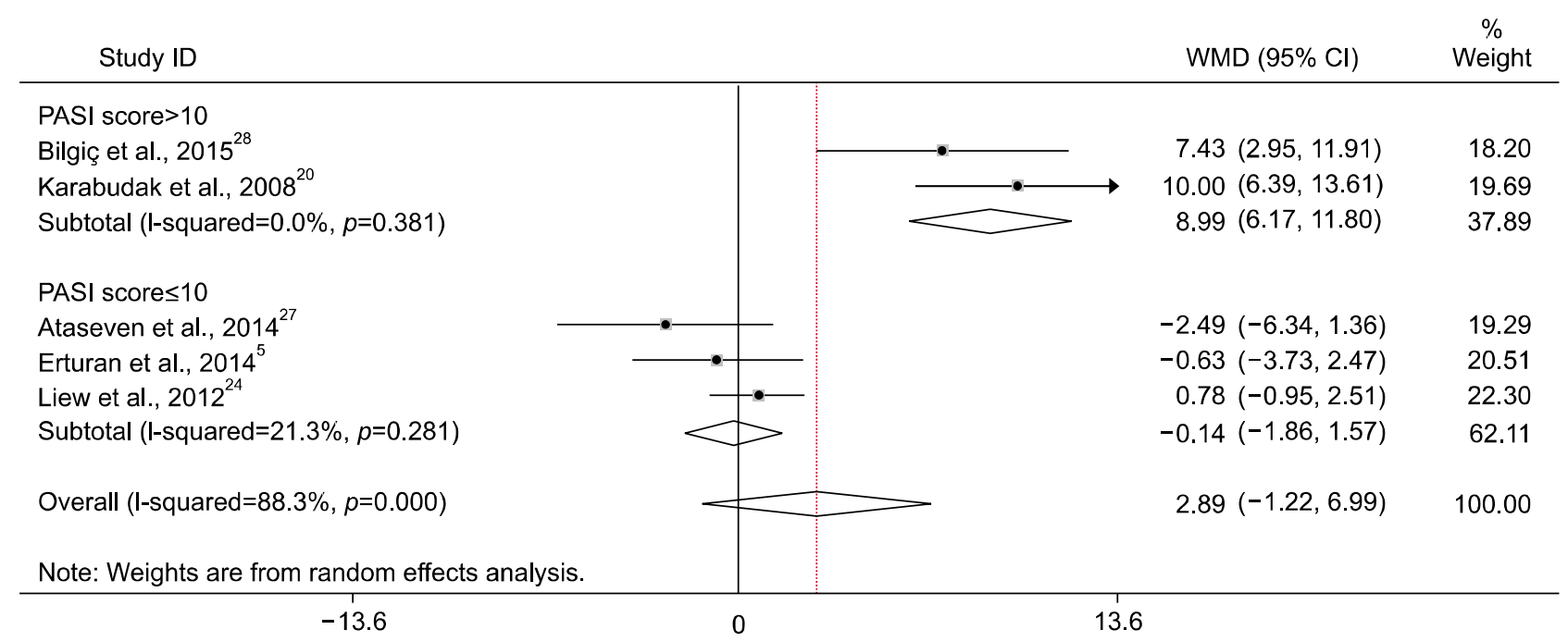

Fig. 3. Forest plot of the meta-analysis for sensitivity analysis. PASI: psoriasis area severity index, WMD: weighted mean difference, $\mathrm{Cl}$ : confidence interval.

lecular damage. This also impacts dendritic cells, T lymphocytes, keratinocytes, angiogenesis, and inflammatory signaling $^{31}$. Hcy has a highly reactive sulfhydryl group, which easily self-oxidizes. More than $98 \%$ of Hcy is in oxidized state. Hcy acts as a pro-oxidant and has been implicated in decreased antioxidant capacity in patients with 


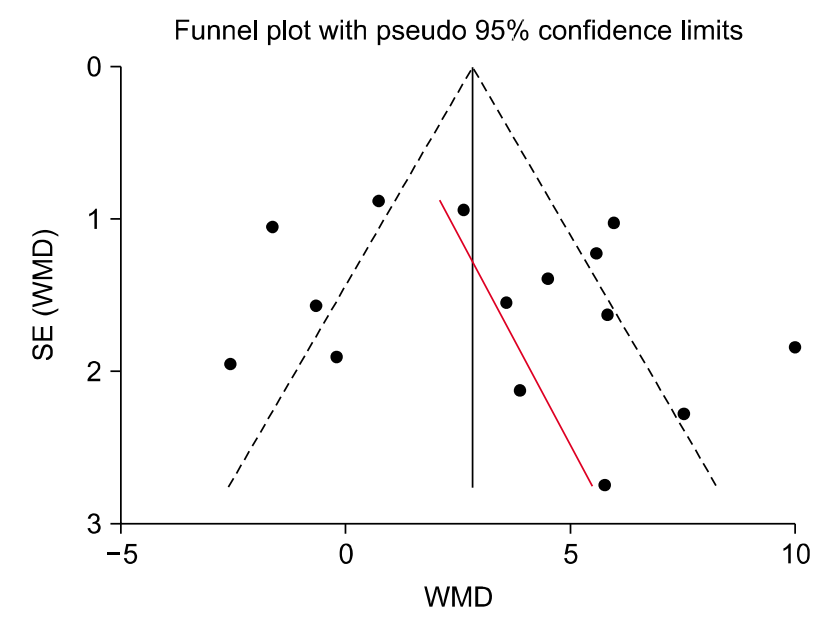

Fig. 4. Funnel plot of included studies. SE: standard error, WMD: weighted mean difference.

inflammatory bowel disease ${ }^{10,32,33}$. In addition, there is a close relationship between CVD and oxidative stress ${ }^{32}$.

Patients with psoriasis have an increased prevalence of CVD compared to the general population ${ }^{34}$. This was originally reported by McDonald and Calabresi ${ }^{35}$, who found that patients with psoriasis had almost twice the incidence of cardiovascular or cerebrovascular disease. Since then, several epidemiological studies have provided evidence supporting the link between psoriasis and $\mathrm{CVD}^{36-38}$ and showing increased prevalence of coronary artery calcification in psoriasis patients ${ }^{39}$. Possible factors underlying the increased risk for CVD in psoriasis patients include hypertension, smoking, excess alcohol intake, dyslipidemia, diabetes mellitus, hyperhomocysteinemia, and inflammation ${ }^{40}$. Elevated levels of Hcy are an independent risk factor for the development of CVD ${ }^{41}$. Hyperhomocysteinemia ( $>15$ $\mu \mathrm{mol} / \mathrm{L}$ ) is associated with increased aortic stiffness, a parameter of the stiffness of central arteries indicative of endothelial dysfunction $^{42}$. In a multicenter European study, Hcy levels $>12 \mu \mathrm{mol} / \mathrm{L}$ were shown to double the risk for CVD, independent of conventional risk factors. The magnitude of the risk for CVD was equivalent to that of smoking or hyperlipidemia ${ }^{41}$. Hcy is believed to cause endothelial dysfunction by causing accumulation of asymmetrical dimethyl arginine, a natural inhibitor of nitric oxide synthase. This results in reduction of the vasodilator nitric oxide which helps protect vessel walls against vascular pathologies, such as atherosclerosis and thrombosis ${ }^{18}$.

Keratinocyte turnover may be accelerated in patients with psoriasis, and folate, used to methylate DNA in actively dividing cells, may be consumed, leading to higher levels of $\mathrm{Hcy}^{43-45}$. Psoriasis patients often present with low levels of folic acid as a result of increased vitamin utilization in the skin and/or reduced gut absorption ${ }^{15,18}$. As dietary nu- trients such as folic acid, vitamin B6, and vitamin B12 are all involved in Hcy metabolism in the blood, supplementation with these compounds is recommended in psoriasis patients ${ }^{14}$.

In our meta-analysis, Hcy levels were significantly higher in psoriasis patients than in healthy controls. Furthermore, subgroup and sensitivity analyses revealed that patients with psoriasis showed significantly higher Hcy levels compared with controls only in studies with high mean PASI score (PASI > 10). In addition, meta-analysis of two studies $^{12,29}$ which separately evaluated Hcy levels based on psoriasis severity showed that patients with moderate-severe psoriasis had significantly higher Hcy levels compared to those with mild psoriasis.

This study had several limitations that should be considered. First, all studies included in this meta-analysis were retrospective studies, and did not include blinding or randomization, which may have resulted in bias. Second, although Egger's test showed no evidence of significant publication bias, but possible publication bias was still existed in our meta-analysis. There could be unpublished negative studies or incomplete raw data which might influence the results. Third, the geographic regions were limited to Turkey, Italy, Malaysia, Norway, and Spain, and thus our results may not be indicative of other populations. Fourth, the sample size was small and there was heterogeneity existed in the eligible studies. Lastly, plasma levels of Hcy can be influenced by a variety of other factors including age, sex, and systemic disease, which was not controlled for in all studies. And also the positive associations of psoriasis and serum Hcy level which was shown in this study may not be due to psoriasis itself and may be due to CVDs that can concurrently occur in psoriasis. Additional well-designed longitudinal epidemiological studies with larger sample sizes representing a more diverse patient population are required. Such studies may be used to determine whether serum levels of Hcy can be used as a predictive and prognostic marker for disease severity in psoriasis patients.

Although our study confirmed elevated levels of Hcy are significantly associated with psoriasis, particularly in patients with a mean PASI score greater than 10, the pathophysiological connections are complex and remain unclear. It is likely that these changes involve mechanisms that underlie chronic inflammatory conditions of psoriasis including inflammatory cytokines, and metabolic, immune, and endocrine changes. Also, higher plasma levels of Hcy are likely an important factor underpinning increased cardiovascular comorbidities in patients with psoriasis. Future studies are needed to investigate the pathogenetic mechanisms of Hcy on psoriasis and association between Hcy 
levels and psoriasis severity.

\section{ACKNOWLEDGMENT}

This work was supported by the National Research Foundation of Korea (NRF-2018R1C1B5042271) and the Soonchunhyang University Research Fund.

\section{SUPPLEMENTARY MATERIALS}

Supplementary data can be found via http://anndermatol. org/src/sm/ad-31-378-s001.pdf.

\section{CONFLICTS OF INTEREST}

The authors have nothing to disclose.

\section{ORCID}

Jung Eun Kim, https://orcid.org/0000-0002-8399-8456

Ho Jung Lee, https://orcid.org/0000-0001-9674-6429

Jong Suk Lee, https://orcid.org/0000-0002-6554-7598

Kyu Uang Whang, https://orcid.org/0000-0002-5483-8410

Young Lip Park, https://orcid.org/0000-0002-6532-3156

Sung Yul Lee, https://orcid.org/0000-0002-6995-4561

Hyun Jung Kim, https://orcid.org/0000-0003-2018-2385

\section{REFERENCES}

1. Neimann AL, Shin DB, Wang $X$, Margolis DJ, Troxel AB, Gelfand JM. Prevalence of cardiovascular risk factors in patients with psoriasis. J Am Acad Dermatol 2006;55:829835.

2. Arias-Santiago S, Orgaz-Molina J, Castellote-Caballero L, Arrabal-Polo MÁ, García-Rodriguez S, Perandrés-López R, et al. Atheroma plaque, metabolic syndrome and inflammation in patients with psoriasis. Eur J Dermatol 2012;22: 337-344.

3. Vena GA, Altomare G, Ayala F, Berardesca E, CalzavaraPinton $\mathrm{P}$, Chimenti $\mathrm{S}$, et al. Incidence of psoriasis and association with comorbidities in Italy: a 5-year observational study from a national primary care database. Eur J Dermatol 2010;20:593-598.

4. Reich K. The concept of psoriasis as a systemic inflammation: implications for disease management. J Eur Acad Dermatol Venereol 2012;26 Suppl 2:3-11.

5. Erturan I, Köroğlu BK, Adiloğlu A, Ceyhan AM, Akkaya VB, Tamer $\mathrm{N}$, et al. Evaluation of serum SCD40L and homocysteine levels with subclinical atherosclerosis indicators in patients with psoriasis: a pilot study. Int J Dermatol 2014; 53:503-509.

6. Ahlehoff $\mathrm{O}$, Gislason $\mathrm{GH}$, Charlot $\mathrm{M}$, Jørgensen $\mathrm{CH}$, Lindhardsen J, Olesen JB, et al. Psoriasis is associated with clinically significant cardiovascular risk: a Danish nationwide cohort study. J Intern Med 2011;270:147-157.

7. Hjuler KF, Gormsen LC, Vendelbo MH, Egeberg A, Nielsen J, Iversen L. Increased global arterial and subcutaneous adipose tissue inflammation in patients with moderate-tosevere psoriasis. Br J Dermatol 2017;176:732-740.

8. Dowlatshahi EA, van der Voort EA, Arends LR, Nijsten T. Markers of systemic inflammation in psoriasis: a systematic review and meta-analysis. Br J Dermatol 2013;169:266-282.

9. Davidovici BB, Sattar N, Prinz J, Puig L, Emery P, Barker JN, et al. Psoriasis and systemic inflammatory diseases: potential mechanistic links between skin disease and co-morbid conditions. J Invest Dermatol 2010;130:1785-1796.

10. Oussalah A, Guéant JL, Peyrin-Biroulet L. Meta-analysis: hyperhomocysteinaemia in inflammatory bowel diseases. Aliment Pharmacol Ther 2011;34:1173-1184.

11. Wang WM, Jin HZ. Homocysteine: a potential common route for cardiovascular risk and DNA methylation in psoriasis. Chin Med J (Engl) 2017;130:1980-1986.

12. Brazzelli V, Grasso V, Fornara L, Moggio E, Gamba G, Villani S, et al. Homocysteine, vitamin B12 and folic acid levels in psoriatic patients and correlation with disease severity. Int J Immunopathol Pharmacol 2010;23:911-916.

13. Giannoni M, Consales V, Campanati A, Ganzetti G, Giuliodori K, Postacchini V, et al. Homocysteine plasma levels in psoriasis patients: our experience and review of the literature. J Eur Acad Dermatol Venereol 2015;29:17811785.

14. Richetta AG, Mattozzi C, Macaluso L, Cantisani C, Giancristoforo S, D'epiro S, et al. Homocysteine plasmatic status in patients with psoriasis. Eur J Dermatol 2011;21:621-623.

15. Malerba M, Gisondi $P$, Radaeli A, Sala R, Calzavara Pinton PG, Girolomoni G. Plasma homocysteine and folate levels in patients with chronic plaque psoriasis. $\mathrm{Br} \mathrm{J}$ Dermatol 2006;155:1165-1169.

16. Vanizor Kural B, Orem A, Cimşit G, Uydu HA, Yandi YE, Alver A. Plasma homocysteine and its relationships with atherothrombotic markers in psoriatic patients. Clin Chim Acta 2003;332:23-30.

17. Refsum H, Helland S, Ueland PM. Fasting plasma homocysteine as a sensitive parameter of antifolate effect: a study of psoriasis patients receiving low-dose methotrexate treatment. Clin Pharmacol Ther 1989;46:510-520.

18. Tobin AM, Hughes R, Hand EB, Leong T, Graham IM, Kirby $B$. Homocysteine status and cardiovascular risk factors in patients with psoriasis: a case-control study. Clin Exp Dermatol 2011;36:19-23.

19. Moher D, Liberati A, Tetzlaff J, Altman DG; and the PRISMA Group. Preferred reporting items for systematic reviews and meta-analysis: the PRISMA Statment. Ann Intern Med 2009;151:264-269.

20. Karabudak O, Ulusoy RE, Erikci AA, Solmazgul E, Dogan B, Harmanyeri Y. Inflammation and hypercoagulable state in adult psoriatic men. Acta Derm Venereol 2008;88:337-340.

21. Cakmak SK, Gül U, Kiliç C, Gönül M, Soylu S, Kiliç A. Homocysteine, vitamin B12 and folic acid levels in psoriasis patients. J Eur Acad Dermatol Venereol 2009;23:300-303. 
22. Dogan S, Atakan N. Is serum amyloid A protein a better indicator of inflammation in severe psoriasis? $\mathrm{Br} J$ Dermatol 2010;163:895-896.

23. Gisondi P, Malerba M, Malara G, Puglisi Guerra A, Sala R, Radaeli $A$, et al. C-reactive protein and markers for thrombophilia in patients with chronic plaque psoriasis. Int J Immunopathol Pharmacol 2010;23:1195-1202.

24. Liew SC, Das-Gupta E, Wong SF, Lee N, Safdar N, Jamil A. Association of methylentetraydrofolate reductase (MTHFR) $677 \mathrm{C}>\mathrm{T}$ gene polymorphism and homocysteine levels in psoriasis vulgaris patients from Malaysia: a case-control study. Nutr J 2012;11:1.

25. Romaní J, Caixàs $A$, Carrascosa JM, Ribera $M$, Rigla $M$, Luelmo J. Effect of narrowband ultraviolet B therapy on inflammatory markers and body fat composition in moderate to severe psoriasis. Br J Dermatol 2012;166:1237-1244.

26. Akcali C, Buyukcelik B, Kirtak N, Inaloz S. Clinical and laboratory parameters associated with metabolic syndrome in Turkish patients with psoriasis. J Int Med Res 2014;42: 386-394.

27. Ataseven A, Kesli R, Kurtipek GS, Ozturk P. Assessment of lipocalin 2, clusterin, soluble tumor necrosis factor receptor-1, interleukin-6, homocysteine, and uric acid levels in patients with psoriasis. Dis Markers 2014;2014:541709.

28. Bilgiç Ö, Altınyazar HC, Baran $H$, Ünlü A. Serum homocysteine, asymmetric dimethyl arginine (ADMA) and other arginine-NO pathway metabolite levels in patients with psoriasis. Arch Dermatol Res 2015;307:439-444.

29. Curcó N, Barriendos N, Barahona MJ, Arteaga C, García M, Yordanov $\mathrm{S}$, et al. Factors influencing cardiometabolic risk profile in patients with psoriasis. Australas J Dermatol 2018; 59:e93-e98.

30. Hayden JA, van der Windt DA, Cartwright JL, Côté P, Bombardier C. Assessing bias in studies of prognostic factors. Ann Intern Med 2013;158:280-286.

31. Lin X, Huang T. Oxidative stress in psoriasis and potential therapeutic use of antioxidants. Free Radic Res 2016;50: 585-595.

32. Mansoor MA, Bergmark C, Svardal AM, Lønning PE, Ueland PM. Redox status and protein binding of plasma homocysteine and other aminothiols in patients with earlyonset peripheral vascular disease. Homocysteine and peri- pheral vascular disease. Arterioscler Thromb Vasc Biol 1995; 15:232-240.

33. Koutroubakis IE, Malliaraki N, Dimoulios PD, Karmiris K, Castanas E, Kouroumalis EA. Decreased total and corrected antioxidant capacity in patients with inflammatory bowel disease. Dig Dis Sci 2004;49:1433-1437.

34. Pearce DJ, Morrison AE, Higgins KB, Crane MM, Balkrishnan $R$, Fleischer $A B$ Jr, et al. The comorbid state of psoriasis patients in a university dermatology practice. J Dermatolog Treat 2005;16:319-323.

35. McDonald CJ, Calabresi P. Thromboembolic disorders associated with psoriasis. Arch Dermatol 1973;107:918.

36. Lindegård B. Diseases associated with psoriasis in a general population of 159,200 middle-aged, urban, native Swedes. Dermatologica 1986;172:298-304.

37. Henseler T, Christophers E. Disease concomitance in psoriasis. J Am Acad Dermatol 1995;32:982-986.

38. Gelfand JM, Neimann AL, Shin DB, Wang X, Margolis DJ, Troxel AB. Risk of myocardial infarction in patients with psoriasis. JAMA 2006;296:1735-1741.

39. Ludwig RJ, Herzog C, Rostock A, Ochsendorf FR, Zollner TM, Thaci $D$, et al. Psoriasis: a possible risk factor for development of coronary artery calcification. $\mathrm{Br} J$ Dermatol 2007; 156:271-276.

40. Kremers HM, McEvoy MT, Dann FJ, Gabriel SE. Heart disease in psoriasis. J Am Acad Dermatol 2007;57:347-354.

41. Graham IM, Daly LE, Refsum HM, Robinson K, Brattström LE, Ueland PM, et al. Plasma homocysteine as a risk factor for vascular disease. The European Concerted Action Project. JAMA 1997;277:1775-1781.

42. Mayer O, Filipovský J, Dolejsová M, Cífková R, Simon J, Bolek L. Mild hyperhomocysteinaemia is associated with increased aortic stiffness in general population. J Hum Hypertens 2006;20:267-271.

43. Hild DH. Folate losses from the skin in exfoliative dermatitis. Arch Intern Med 1969;123:51-57.

44. Fry L, Macdonald A, Almeyda J, Griffin CJ, Hoffbrand AV. The mechanism of folate deficiency in psoriasis. $\mathrm{Br}$ J Dermatol 1971;84:539-544.

45. Touraine R, Revuz J, Zittoun J, Jarret J, Tulliez M. Study of folate in psoriasis: blood levels, intestinal absorption and cutaneous loss. Br J Dermatol 1973;89:335-341. 